\title{
Scalable, Catalytic Asymmetric Synthesis of Syn, Anti Stereotriad Building Blocks for Polypropionate Antibiotics
}

\author{
Kathlyn A. Parker* and Huanyan Cao \\ Department of Chemistry \\ State University of New York at Stony Brook \\ Stony Brook, New York 11794-3400 \\ kparker@notes.cc.sunysb.edu
}

\section{Supporting Information \#1 - Experimental Procedures}

Contents

page in Supporting Information \#1

General Experimental Protocols....................... 2

Chiral alcohol (S)-11............................... 2

Mosher ester of chiral alcohol (S)-11................. 3

Racemic alcohol 11................................ 3

Mosher ester of racemic alcohol 11...................... 3

Ether 12......................................... 4

Alcohol 13........................................ 4

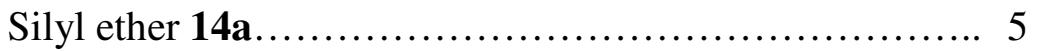

MOM ether 14b..................................... 5

Alcohol 8a.......................................... 6

Alcohol 8b........................................ 6

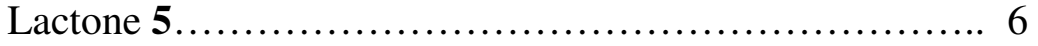

Methoxybenzyl ether 16............................... 7

Aldehyde 17........................................ 7

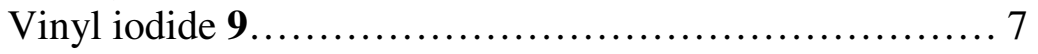




\section{General Information}

Unless noted otherwise, all oxygen and moisture-sensitive reactions were executed in oven-dried glassware sealed under a positive pressure of dry argon and moisture sensitive solutions and anhydrous solvents were transferred via standard syringe and cannula techniques. Unless stated otherwise, all commercial reagents, were used as received. All solvents were dried under argon atmosphere: THF and diethyl ether were distilled over Na-benzophenone; $\mathrm{CH}_{2} \mathrm{Cl}_{2}$, were distilled from $\mathrm{CaH}_{2}$. The extracts were dried over $\mathrm{Na}_{2} \mathrm{SO}_{4}$ unless otherwise noted. Flash chromatography was performed using Scientific Flash silica gel ( $32-63 \mathrm{mM}$ ); analytical TLC was performed using $0.25 \mathrm{~mm}$ EM silica gel 60 F254 plates that were visualized by irradiation $(254 \mathrm{~nm})$ or by staining $10 \%$ PMA. IR spectra were recorded using a Galaxy Series FTIR 3000 infrared spectrophotometer. NMR spectra were obtained using Gemini-300MHz (Varian) and Inova-400 (Varian) $\mathrm{MHz}$ instruments.

\section{Experimental Procedures}

Chiral alcohol (S)-11.

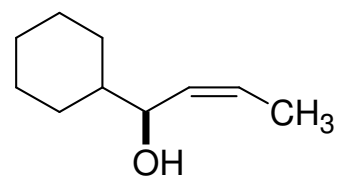

11

To the lithium powder $(562 \mathrm{mg}, 81 \mathrm{mmol})$ under argon was added dry ether $(50 \mathrm{~mL})$ and the suspension was cooled to $-35^{\circ} \mathrm{C}$. With stirring, a solution of (Z)-1-bromo-propene (4.84 g, $40 \mathrm{mmol}$ ) was added dropwise. The mixture was stirred at $-35^{\circ} \mathrm{C}$ for $2 \mathrm{~h}$ and treated dropwise with zinc bromide solution $(0.6 \mathrm{M}$ in ether, $77 \mathrm{~mL}, 44 \mathrm{mmol})$. The reaction mixture was stirred for an additional $1 \mathrm{~h}$ at $0{ }^{\circ} \mathrm{C}$ and then a solution of lithium $(1 \mathrm{R}, 2 \mathrm{~S})-\mathrm{N}$-methylephedrate, prepared by the addition of $\mathrm{n}$-butyllithium $(2.5 \mathrm{M}$ in hexanes, $16.4 \mathrm{~mL}, 41 \mathrm{mmol})$ to a solution of (-)-N-methylephedrine $(7.35 \mathrm{~g}, 41 \mathrm{mmol})$ in toluene $(100 \mathrm{~mL})$ at $0{ }^{\circ} \mathrm{C}$, was added by cannula. The solution was stirred for $1 \mathrm{~h}$ at $0{ }^{\circ} \mathrm{C}$ and cyclohexanecarboxaldehyde $(3.20 \mathrm{~g}, 28.6 \mathrm{mmol})$ was added neat. After stirring for $1 \mathrm{~h}$ at $0{ }^{\circ} \mathrm{C}$, the reaction was quenched by the addition of saturated aqueous ammonium chloride solution. The organic phase was separated and the aqueous phase was extracted with ether. The organic phase was washed with a second portion of the ammonium chloride solution, dried $\left(\mathrm{MgSO}_{4}\right)$, and concentrated. Chromatography (HE: EA = 10: 1) gave alcohol 11 (3.61 g, 82\%, 92\% ee. according to NMR study of Mosher ester) as clear liquid. (-)-N-methylephedrine was recovered quantitatively from the aqueous phases by basification with $\mathrm{NaOH}$ solution $(\mathrm{pH}=12)$ and extraction into ether. ${ }^{1} \mathrm{H}$ NMR $(300 \mathrm{MHz}$, $\left.\mathrm{CDCl}_{3}\right) \delta 5.57(\mathrm{dqd}, \mathrm{J}=10.2,6.8,1.3 \mathrm{~Hz}, 1 \mathrm{H}), 5.36(\mathrm{ddq}, \mathrm{J}=10.2,9.4,1.8 \mathrm{~Hz}, 1 \mathrm{H}), 4.14$ $(\mathrm{dd}, \mathrm{J}=8.4 \mathrm{~Hz}, 1.0 \mathrm{~Hz}, 1 \mathrm{H}), 1.90(\mathrm{~m}, 1 \mathrm{H}), 1.80-1.58(\mathrm{~m}, 8 \mathrm{H}), 1.58-0.80(\mathrm{~m}, 6 \mathrm{H}) ;{ }^{13} \mathrm{C}$ NMR (75 MHz, $\left.\mathrm{CDCl}_{3}\right) \delta 132.4,126.9,71.7,44.3,29.0,28.6,26.8,26.3,26.2,13.7$; IR (neat) $v_{\max } 3365$ (broad), 2923, 2852, 1449, 1022, 994. 


\section{Mosher ester of chiral alcohol (S-)11}

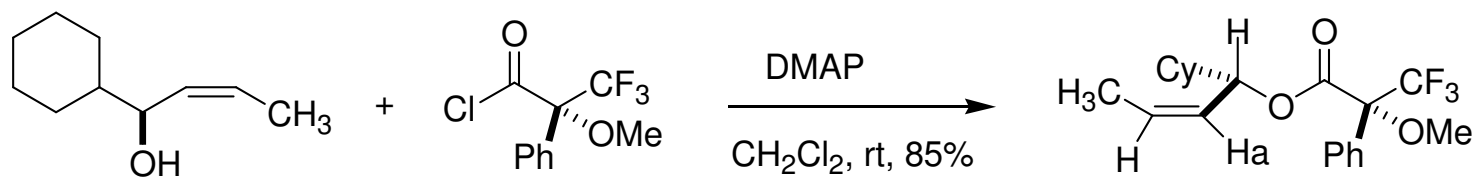

Chiral alcohol 11 (15.4 mg, $0.10 \mathrm{mmol}$ ) and DMAP (54.9 mg, $0.60 \mathrm{mmol}$ ) was dissolved in $\mathrm{CH}_{2} \mathrm{Cl}_{2}(1 \mathrm{Ml})$ and $S$ - $\alpha$-methoxy- $\alpha$-trifluoromethyl phenyl acetyl chloride $(37.4 \mu \mathrm{L}$, $0.20 \mathrm{mmol})$ was added dropwise. After $2 \mathrm{~h}$ at room temperature, water $(5 \mathrm{~mL})$ and EtOAc $(15 \mathrm{~mL})$ were added. The organic phase was washed with saturated $\mathrm{NaHCO}_{3}(2 \times 5 \mathrm{~mL})$. The organic phase was isolated, dried $\left(\mathrm{MgSO}_{4}\right)$, and concentrated. The residue was dissolved and washed with solvent $(\mathrm{HE}: \mathrm{EA}=10: 1)$ through a silica gel column to give crude Mosher ester of chiral alcohol 11 as a colorless oil $(31.5 \mathrm{mg}, 85 \%)$ for ${ }^{1} \mathrm{H}$ NMR analysis. ${ }^{1} \mathrm{H}$ NMR for $\mathrm{Ha}\left(300 \mathrm{MHz}, \mathrm{CDCl}_{3}\right) \delta 5.23(\mathrm{ddq}, \mathrm{J}=10.2,9.6,1.5 \mathrm{~Hz}, 0.96 \mathrm{H})$, 5.38 (ddq, $\mathrm{J}=10.2,9.6,1.5 \mathrm{~Hz}, 0.04 \mathrm{H}$ ).

\section{Racemic alcohol 11.}<smiles>O=CC1CCCCC1</smiles>
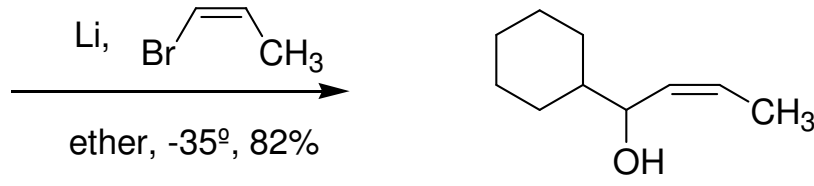

Racemic alcohol 11 was synthesized by the procedure for the preparation of chiral alcohol 11 except that no chiral ligand was added.

\section{Mosher ester of racemic alcohol 11.}<smiles>C/C=C/[C@H](O)C1CCCCC1</smiles><smiles>COC(C(=O)Cl)(c1ccccc1)C(F)(F)F</smiles>

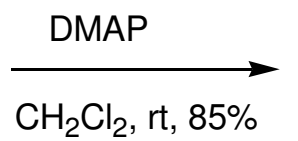<smiles>CC=C[C@@H](Cl)OC(=O)C(Cl)(OC)c1ccccc1</smiles>

Mosher ester of racemic alcohol 11 was synthesized by the same procedure as that used for the preparation of the Mosher ester of chiral alcohol 11. ${ }^{1} \mathrm{H}$ NMR for Ha $(300 \mathrm{MHz}$, $\left.\mathrm{CDCl}_{3}\right) \delta 5.23(\mathrm{ddq}, \mathrm{J}=10.2,9.6,1.5 \mathrm{~Hz}, 0.50 \mathrm{H}), 5.38(\mathrm{ddq}, \mathrm{J}=10.2,9.6,1.5 \mathrm{~Hz}, 0.50$ $\mathrm{H})$. 


\section{Ether 12.}

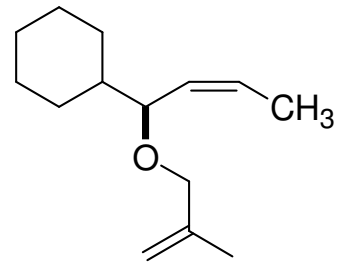

12

A $100-\mathrm{mL}$ reaction flask was charged with $95 \%$ sodium hydride $(2.76 \mathrm{~g}, 109 \mathrm{mmol})$ and $20 \mathrm{~mL}$ of dry THF. Alcohol $11(2.40 \mathrm{~g}, 15.6 \mathrm{mmol})$ in $2 \mathrm{~mL}$ of THF was added dropwise followed by 3-chloro-2-methylpropene $(4.24 \mathrm{~g}, 46.8 \mathrm{mmol})$. The reaction mixture refluxed overnight and cooled to room temperature. $3 \mathrm{~mL}$ of water was added slowly to quench the excess sodium hydride. The mixture was poured into water and extracted with ether $(30 \mathrm{~mL} \times 3)$. The combined organic layers were dried over $\mathrm{MgSO}_{4}$, filtered, and concentrated. Chromatography (HE: EA = 30: 1) produced compound $12(2.78 \mathrm{~g}, 85 \%)$ as colorless oil. ${ }^{1} \mathrm{H}$ NMR $\left(300 \mathrm{MHz}, \mathrm{CDCl}_{3}\right) \delta 5.73(\mathrm{dqd}, \mathrm{J}=10.2,6.8,1.3 \mathrm{~Hz}, 1 \mathrm{H}), 5.24$ (ddq, 10.2, 9.4, $1.8 \mathrm{~Hz}, 1 \mathrm{H}), 4.93(\mathrm{~m}, 1 \mathrm{H}), 4.85(\mathrm{~m}, 1 \mathrm{H}), 3.90(\mathrm{~d}, \mathrm{~J}=12.6 \mathrm{~Hz}, 1 \mathrm{H}), 3.80$ $(\mathrm{m}, 1 \mathrm{H}), 3.69(\mathrm{~d}, \mathrm{~J}=12.4 \mathrm{~Hz}, 1 \mathrm{H}), 1.96(\mathrm{~m}, 1 \mathrm{H}), 1.73(\mathrm{~s}, 3 \mathrm{H}), 1.70-1.65(\mathrm{~m}, 5 \mathrm{H}), 1.62$ $(\mathrm{dd}, \mathrm{J}=7.1,2.0 \mathrm{~Hz}, 3 \mathrm{H}), 1.58-0.80(\mathrm{~m}, 6 \mathrm{H}) ;{ }^{13} \mathrm{C} \mathrm{NMR}\left(75 \mathrm{MHz}, \mathrm{CDCl}_{3}\right) \delta 143.3,130.9$, 128.1, 112.1, 77.9, 72.0, 43.1, 31.9, 29.7, 28.9, 27.0, 26.5, 26.4, 23.0, 20.0, 14.4, 13.9; IR (neat) $v_{\max }$ 2971, 2922, 2852, 1449, 1085, 896; HRMS (EI) calcd for $\mathrm{C}_{14} \mathrm{H}_{24} \mathrm{O}\left(\mathrm{M}^{+}\right)$ 208.1827, found 208.1821.

Alcohol 13.

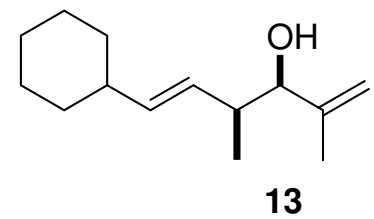

Potassium tert-butoxide (1.0 M in THF, $5.5 \mathrm{~mL}, 5.5 \mathrm{mmol}$ ) was added to a flask under argon and an additional $5.0 \mathrm{~mL}$ of $\mathrm{THF}$ was added. The solution was cooled to $-78^{\circ} \mathrm{C}$ and ether 12 (5.0 mmol, $1.05 \mathrm{~g}$ ) was added. $n$-Butyllithium (1.6M in THF, $3.8 \mathrm{~mL}, 6.1$ mmol) was slowly added. The mixture was warmed to $-20^{\circ} \mathrm{C}$ over $4 \mathrm{~h}$ and the stirred 12 $\mathrm{h}$ at $-20{ }^{\circ} \mathrm{C}$ and $2 \mathrm{~h}$ at $0{ }^{\circ} \mathrm{C}$. The reaction was quenched with water and then extracted with ether. The organic phase was dried over $\mathrm{MgSO}_{4}$, filtered and concentrated. Chromatography (HE: EA = 10:1) gave alcohol $13(819 \mathrm{mg}, 78 \%)$ as colorless oil. ${ }^{1} \mathrm{H}$ NMR $\left(300 \mathrm{MHz}, \mathrm{CDCl}_{3}\right) \delta 5.40(\mathrm{dd}, \mathrm{J}=16.2,6.6 \mathrm{~Hz}, 1 \mathrm{H}), 5.31(\mathrm{dd}, \mathrm{J}=16.2,6.6 \mathrm{~Hz}$, $1 \mathrm{H}), 4.92(\mathrm{~m}, 1 \mathrm{H}), 4.86(\mathrm{~m}, 1 \mathrm{H}), 3.87(\mathrm{~d}, \mathrm{~J}=6.0 \mathrm{~Hz}, 1 \mathrm{H}), 2.33(\mathrm{~m}, 1 \mathrm{H}), 1.90(\mathrm{~m}, 1 \mathrm{H})$, $1.69(\mathrm{~s}, 3 \mathrm{H}), 1.68-1.65(\mathrm{~m}, 5 \mathrm{H}), 1.32-1.05(\mathrm{~m}, 6 \mathrm{H}), 0.97(\mathrm{~d}, \mathrm{~J}=6.9 \mathrm{~Hz}, 3 \mathrm{H}) ;{ }^{13} \mathrm{C} \mathrm{NMR}$ $\left(75 \mathrm{MHz}, \mathrm{CDCl}_{3}\right) \delta 146.2,137.4,129.9,112.1,79.3,41.0,40.1,33.45,33.43,26.5,26.4$, 18.9, 14.8; IR (neat) $v_{\max } 3403$ (broad), 2965, 2924, 2851, 1448, 979, 968, 896; HRMS (EI) calcd for $\mathrm{C}_{14} \mathrm{H}_{24} \mathrm{O}\left(\mathrm{M}^{+}\right)$208.1827, found 208.1821. 
TBS ether 14a.

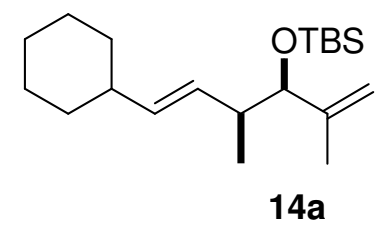

A mixture of alcohol $13(1.27 \mathrm{~g}, 6.13 \mathrm{mmol})$ and 2, 6-lutidine $(1.15 \mathrm{~g}, 1.25 \mathrm{~mL}, 10.7$ $\mathrm{mmol})$ in $20 \mathrm{~mL}$ of methylene chloride was cooled to $-20{ }^{\circ} \mathrm{C}$. $t$-BuMe $\mathrm{BSiOTf}_{2}(1.86 \mathrm{~g}$, $1.62 \mathrm{~mL}, 7.05 \mathrm{mmol}$ ) was added over $5 \mathrm{~min}$. The mixture was stirred for $1 \mathrm{~h}$ at $-20^{\circ} \mathrm{C}$ and $30 \mathrm{~min}$ at room temperature. The mixture was diluted with $40 \mathrm{~mL}$ of ether and poured into $25 \mathrm{~mL}$ of $1 \mathrm{M} \mathrm{NaHSO}_{4}$ solution. The resulting layers were separated and water phase was extracted with ether. The combined organic solution was washed with 1 $\mathrm{M} \mathrm{NaHSO}_{4}$ solution, sodium bicarbonate and brine. The solution was then dried over $\mathrm{MgSO}_{4}$, filtered, and concentrated. Chromatography (HE: EA = 30: 1) gave 14a as a colorless oil $(1.88 \mathrm{~g}, 95 \%) .{ }^{1} \mathrm{H}$ NMR $\left(300 \mathrm{MHz}, \mathrm{CDCl}_{3}\right) \delta 5.30(\mathrm{dd}, \mathrm{J}=16.1,6.6 \mathrm{~Hz}$, $1 \mathrm{H}), 5.19(\mathrm{dd}, \mathrm{J}=16.1,7.5 \mathrm{~Hz}, 1 \mathrm{H}), 4.78(\mathrm{~m}, 1 \mathrm{H}), 4.75(\mathrm{~m}, 1 \mathrm{H}), 3.71(\mathrm{~d}, \mathrm{~J}=7.0 \mathrm{~Hz}, 1 \mathrm{H})$, $2.17(\mathrm{~m}, 1 \mathrm{H}), 1.88(\mathrm{~m}, 1 \mathrm{H}), 1.75-1.64(\mathrm{~m}, 4 \mathrm{H}), 1.63(\mathrm{~s}, 3 \mathrm{H}), 1.32-0.96(\mathrm{~m}, 6 \mathrm{H}), 0.94(\mathrm{~d}, \mathrm{~J}$ $=7.0 \mathrm{~Hz}, 3 \mathrm{H}), 0.89(\mathrm{~s}, 9 \mathrm{H}), 0.02(\mathrm{~s}, 3 \mathrm{H}),-0.03(\mathrm{~s}, 3 \mathrm{H}) ;{ }^{13} \mathrm{C} \mathrm{NMR}\left(75 \mathrm{MHz}, \mathrm{CDCl}_{3}\right) \delta$ $146.9,135.7,130.8,112.1,81.5,41.3,41.0,33.43,33.37,26.6,26.4,26.2$, 18.6, 18.1, 16.4, -4.3, -4.7; IR (neat) $v_{\max } 2957,2927,2854,1449,1251,1071,896,867$; HRMS (FAB) calcd for $\mathrm{C}_{20} \mathrm{H}_{37} \mathrm{OSi}\left[(\mathrm{M}-\mathrm{H})^{+}\right]$321.2614, found 321.2607.

\section{MOM ether 14b.}

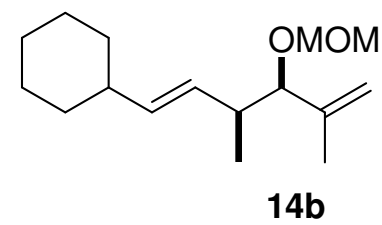

$\mathrm{MOMCl}(567 \mathrm{mg}, 535 \mu \mathrm{L}, 7.05 \mathrm{mmol})$ was added via syringe dropwise to a solution of alcohol 13 (296 mg, $1.41 \mathrm{mmol})$ in $8 \mathrm{~mL}$ of methylene chloride at $0{ }^{\circ} \mathrm{C}$, followed by addition of ${ }^{i} \mathrm{Pr}_{2} \mathrm{NEt}$ (911 mg, $1.23 \mathrm{~mL}, 7.05 \mathrm{mmol}$ ). The resulting mixture was stirred at 0 ${ }^{\circ} \mathrm{C}$ for $2 \mathrm{~h}$ and then at ambient temperature for $16 \mathrm{~h}$. A saturated solution of sodium carbonate $(4 \mathrm{~mL})$ was added to quench the reaction. The aqueous phase was extracted with methylene chloride $(25 \mathrm{~mL}$ x 3$)$ and the organic phases were combined, dried $\left(\mathrm{MgSO}_{4}\right)$, and concentrated. Chromatography $(\mathrm{HE}: \mathrm{EA}=10: 1)$ gave ether $\mathbf{1 4 b}$ as a colorless oil $(323 \mathrm{mg}, 90 \%) .{ }^{1} \mathrm{H}$ NMR $\left(300 \mathrm{MHz}, \mathrm{CDCl}_{3}\right) \delta 5.35(\mathrm{dd}, \mathrm{J}=15.6,6.6 \mathrm{~Hz}$, $1 \mathrm{H}), 5.17(\mathrm{dd}, \mathrm{J}=12.6,7.8 \mathrm{~Hz}, 1 \mathrm{H}), 4.91(\mathrm{~d}, \mathrm{~J}=0.9 \mathrm{~Hz}, 1 \mathrm{H}), 4.84(\mathrm{~s}, 1 \mathrm{H}), 4.61(\mathrm{~m}, 1 \mathrm{H})$, $4.47(\mathrm{~d}, \mathrm{~J}=6.6 \mathrm{~Hz}, 1 \mathrm{H}), 3.67(\mathrm{~d}, \mathrm{~J}=7.2 \mathrm{~Hz}, 1 \mathrm{H}), 3.37(\mathrm{~s}, 1 \mathrm{H}), 2.27(\mathrm{~m}, 1 \mathrm{H}), 1.86(\mathrm{~m}$, $1 \mathrm{H}), 1.75-1.45(\mathrm{~m}, 7 \mathrm{H}), 0.90-1.30(\mathrm{~m}, 9 \mathrm{H}) ;{ }^{13} \mathrm{C} \mathrm{NMR}\left(75 \mathrm{MHz}, \mathrm{CDCl}_{3}\right) \delta 143.2,136.2$, $129.8,115.3,93.9,84.7,55.9,40.9,39.5,33.4,26.5,26.3,17.6,17.4$; IR (neat) $v_{\max }$ 2923, 2851, 1450, 1153, 1095, 1033, 968; HRMS (EI) calcd for $\mathrm{C}_{16} \mathrm{H}_{28} \mathrm{O}_{2}\left(\mathrm{M}^{+}\right) 252.2089$, found 252.2083 . 


\section{Alcohol 8a.}

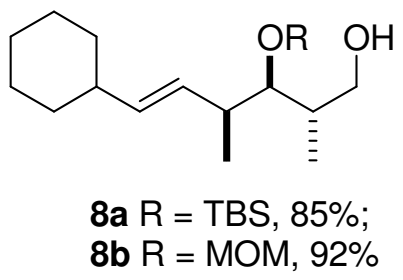

To the silyl ether 14a $(1.57 \mathrm{~g}, 4.84 \mathrm{mmol})$ in $5 \mathrm{~mL}$ of THF was added 9-BBN (0.5 $\mathrm{M}$ in THF, $10.6 \mathrm{~mL}, 5.32 \mathrm{mmol}$ ) at $-20^{\circ} \mathrm{C}$. After $10 \mathrm{~min}$ the reaction mixture was warmed to room temperature and stirring was continued for $6 \mathrm{~h}$. TLC showed the completion of the reaction. Then $3 \mathrm{~mL}$ of $3 \mathrm{~N} \mathrm{NaOH}$ was added and this was followed by $3 \mathrm{~mL}$ of $30 \%$ $\mathrm{H}_{2} \mathrm{O}_{2}$. The resulting mixture was stirred for 12 hours at $\mathrm{rt}$ and then poured into $100 \mathrm{~mL}$ of ether and $20 \mathrm{~mL}$ of saturated aqueous sodium chloride. The aqueous phase was extracted with ether and the organic solutions were combined, dried $\left(\mathrm{MgSO}_{4}\right)$, and concentrated. Chromatography (HE: EA = 10: 1) gave alcohol 8a as a colorless oil $(1.43 \mathrm{~g}, 86 \%) .{ }^{1} \mathrm{H}$ NMR $\left(300 \mathrm{MHz}, \mathrm{CDCl}_{3}\right) \delta 5.36,(\mathrm{~m}, 2 \mathrm{H}), 3.72(\mathrm{dd}, \mathrm{J}=11.0,3.8 \mathrm{~Hz}, 1 \mathrm{H}), 3.52(\mathrm{dd}, \mathrm{J}=$ 11.0, $5.7 \mathrm{~Hz}, 1 \mathrm{H}), 3.49(\mathrm{dd}, \mathrm{J}=6.1,3.9 \mathrm{~Hz}, 1 \mathrm{H}), 2.42-2.30(\mathrm{~m}, 2 \mathrm{H}), 1.96-1.80(\mathrm{~m}, 2 \mathrm{H})$, 1.76-1.58 (m, 5H), 1.32-1.02 (m, 4H), $1.00(\mathrm{~d}, \mathrm{~J}=7.1 \mathrm{~Hz}, 6 \mathrm{H}), 0.92(\mathrm{~s}, 9 \mathrm{H}), 0.11(\mathrm{~s}, 3 \mathrm{H})$, $0.09(\mathrm{~s}, 3 \mathrm{H}) .{ }^{13} \mathrm{C}$ NMR $\left(75 \mathrm{MHz}, \mathrm{CDCl}_{3}\right) \delta 136.9,130.3,81.9,65.9,42.3,41.1,37.5$, $33.37,33.35,26.5,26.4,18.5,17.2,16.7,-3.6,-3.7$; IR (neat) $v_{\max } 3398$ (broad), 2957 , 2927, 1254, 1074, 1024; HRMS (FAB) calcd for $\mathrm{C}_{20} \mathrm{H}_{41} \mathrm{O}_{2} \mathrm{Si}\left[(\mathrm{M}+\mathrm{H})^{+}\right] 341.2876$, found 341.2876 .

Alcohol 8b was prepared by a similar procedure from MOM ether 14b. ${ }^{1} \mathrm{H}$ NMR (400 $\left.\mathrm{MHz} \mathrm{CDCl}_{3}\right) \delta 5.36(\mathrm{~m}, 2 \mathrm{H}), 4.65(\mathrm{~d}, \mathrm{~J}=6.6 \mathrm{~Hz}, 1 \mathrm{H}), 4.59(\mathrm{~d}, \mathrm{~J}=6.6 \mathrm{~Hz}, 1 \mathrm{H}), 3.82$, (m, 1H), 3.49 (m, 1H), 3.41 (s, 3H), 3.34 (dd, J = 7.5, 4.2 Hz, 1H), 2.82 (dd, J = 7.3, $5.7 \mathrm{~Hz}$, $1 \mathrm{H}), 2.37(\mathrm{~m}, 1 \mathrm{H}), 1.95-1.80(\mathrm{~m}, 2 \mathrm{H}), 1.78-1.60(\mathrm{~m}, 5 \mathrm{H}), 1.32-1.00(\mathrm{~m}, 4 \mathrm{H}), 0.98(\mathrm{~d}, \mathrm{~J}=$ $3.7 \mathrm{~Hz}, 3 \mathrm{H}), 0.97(\mathrm{~d}, \mathrm{~J}=3.7 \mathrm{~Hz}, 3 \mathrm{H}) ;{ }^{13} \mathrm{C} \mathrm{NMR}\left(100 \mathrm{MHz}, \mathrm{CDCl}_{3}\right) \delta 136.5,131.0,99.0$, 87.1, 65.4, 56.5, 40.9, 39.3, 37.4, 33.33, 33.27, 26.4, 26.3, 15.3, 14.5; IR (neat) $v_{\max }$ 3420(broad), 2922, 2850, 1449, 1147, 1095, 1032; HRMS (EI) calcd for $\mathrm{C}_{16} \mathrm{H}_{30} \mathrm{O}_{3}\left(\mathrm{M}^{+}\right)$ 270.2195 , found 270.2186 .

\section{Lactone 5.}

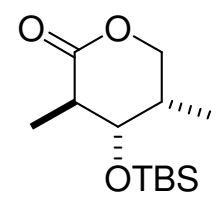

5

A solution of alcohol $8 \mathbf{a}(45 \mathrm{mg}, 0.13 \mathrm{mmol})$ in $\mathrm{CH}_{2} \mathrm{Cl}_{2}(6 \mathrm{~mL})$ cooled to $-78{ }^{\circ} \mathrm{C}$ was flushed with $\mathrm{O}_{3}$. After its color turned to blue, the solution was flushed with argon for 10 min at $-78^{\circ} \mathrm{C}$. Then dimethyl sulfide $(0.5 \mathrm{~mL})$ was added. After $1 \mathrm{~h}$ at $-78^{\circ} \mathrm{C}$ and $6 \mathrm{~h}$ at room temperature, the mixture was concentrated to give a colorless oil. 
The above residue was dissolved in EtOAc $(1 \mathrm{~mL})$ and $\mathrm{MnO}_{2}(229 \mathrm{mg}, 2.6 \mathrm{mmol})$ was added. The mixture was stirred for $48 \mathrm{~h}$ at room temperature and then directly loaded to a silica gel column. Washing with solvent $(\mathrm{HE}: \mathrm{EA}=2: 1$ ) gave a colorless solid, which was crystallized from HE: EA $=3: 1$ to give lactone 5 as a crystalline solid $(23.7 \mathrm{mg}, 80 \%$ for two steps), mp 53-54 ${ }^{\circ} \mathrm{C} ;{ }^{1} \mathrm{H}$ NMR $\left(300 \mathrm{MHz}, \mathrm{CDCl}_{3}\right) \delta 4.26(\mathrm{dd}, \mathrm{J}=11.0,9.6 \mathrm{~Hz}$, $1 \mathrm{H}), 4.11(\mathrm{dd}, \mathrm{J}=11.0,4.9 \mathrm{~Hz}, 1 \mathrm{H}), 3.69$, (t, J = 3.1 Hz, 1H), $2.62(\mathrm{~m}, 1 \mathrm{H}), 2.20(\mathrm{~m}, 1 \mathrm{H})$, $1.30(\mathrm{~d}, \mathrm{~J}=7.4 \mathrm{~Hz}, 3 \mathrm{H}), 0.97(\mathrm{~d}, \mathrm{~J}=6.6 \mathrm{~Hz}, 3 \mathrm{H}), 0.90$ (d, s, 9H), 0.09 (s, 3H), 0.07 (s, $3 \mathrm{H}) ;{ }^{13} \mathrm{C} \mathrm{NMR}\left(100 \mathrm{MHz}, \mathrm{CDCl}_{3}\right) \delta 174.0,73.8,70.3,43.9,30.6,25.9,16.5,12.3,-4.3$, 4.5 .

\section{Methoxybenzyl ether 16.}

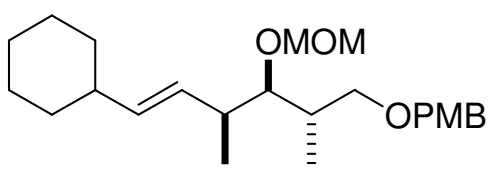

16

To a solution of alcohol $\mathbf{8 b}(136 \mathrm{mg}, 0.50 \mathrm{mmol})$ in DMF $(2 \mathrm{~mL})$ cooled to $0{ }^{\circ} \mathrm{C}$ was added $\mathrm{NaH}(72 \mathrm{mg}, 3.0 \mathrm{mmol})$. After stirring for $30 \mathrm{~min}, \mathrm{Bu}_{4} \mathrm{NI}(18.5 \mathrm{mg}, 0.05 \mathrm{mmol})$ and 4-methoxybenzyl chloride $(100 \mu \mathrm{L}, 0.75 \mathrm{mmol})$ were added successively, and the resulting solution was stirred at room temperature for $18 \mathrm{~h}$. The reaction was quenched by the addition of water $(1 \mathrm{~mL})$, and the mixture was extracted with ether $(5 \times 10 \mathrm{~mL})$. The combined organic layers were dried $\left(\mathrm{MgSO}_{4}\right)$, filtered, and concentrated under reduced pressure. Purification by flash chromatography (HE: EA = $10: 1$ ) afforded ether 16 as a colorless oil (180 mg, 92\%). ${ }^{1} \mathrm{H} \mathrm{NMR}\left(400 \mathrm{MHz}, \mathrm{CDCl}_{3}\right) \delta 7.25(\mathrm{~d}, \mathrm{~J}=8.5 \mathrm{~Hz}$, 2H), $6.87(\mathrm{~d}, \mathrm{~J}=8.5 \mathrm{~Hz}, 2 \mathrm{H}), 5.33(\mathrm{t}, \mathrm{J}=5.6 \mathrm{~Hz}, 1 \mathrm{H}), 4.60(\mathrm{~s}, 2 \mathrm{H}), 4.41(\mathrm{~s}, 2 \mathrm{H}), 3.80(\mathrm{~s}$, $3 \mathrm{H}), 3.54(\mathrm{~m}, 2 \mathrm{H}), 3.36(\mathrm{~s}, 3 \mathrm{H}), 3.32(\mathrm{~m}, 2 \mathrm{H}), 2.35(\mathrm{~m}, 1 \mathrm{H}), 1.99(\mathrm{~m}, 1 \mathrm{H}), 1.88(\mathrm{~m}, 1 \mathrm{H})$, 1.76-1.58 (m, 5H), 1.32-1.02 (m, 4H), $1.00(\mathrm{~d}, \mathrm{~J}=7.1 \mathrm{~Hz}, 3 \mathrm{H}), 0.98(\mathrm{~d}, \mathrm{~J}=7.2 \mathrm{~Hz}, 3 \mathrm{H})$. ${ }^{13} \mathrm{C}$ NMR $\left(75 \mathrm{MHz}, \mathrm{CDCl}_{3}\right) \delta 159.3,136.2,131.22,131.17,129.4,113.9,98.4,85.4$, $72.9,72.4,56.3,55.5,40.9,39.4,36.7,33.31,33.25,26.4,26.3,15.8,15.3$; IR (neat) $v_{\max }$ 2961, 2923, 1513,1248, 1093, 1035; HRMS (FAB) calcd for $\mathrm{C}_{24} \mathrm{H}_{37} \mathrm{O}_{4}\left[(\mathrm{M}-\mathrm{H})^{+}\right]$ 389.2692 , found 389.2698 .

\section{Aldehyde 17.}

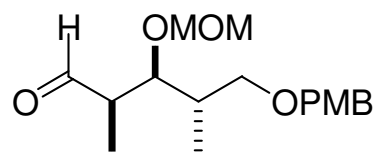

17

To a solution of ether $16(111 \mathrm{mg}, 0.28 \mathrm{mmol})$ in $\mathrm{CH}_{2} \mathrm{Cl}_{2}(10 \mathrm{~mL})$ cooled to $-78{ }^{\circ} \mathrm{C}$ was flushed in $\mathrm{O}_{3}$. After $10 \mathrm{~min}$, the solution color turned into blue. After the solution being flushed with argon for $10 \mathrm{~min}$ at $-78^{\circ} \mathrm{C}, \mathrm{Ph}_{3} \mathrm{P}(89 \mathrm{mg}, 0.30 \mathrm{mmol})$ was added. After $1 \mathrm{~h}$ at $-78^{\circ} \mathrm{C}$ and $6 \mathrm{~h}$ at room temperature, the mixture was concentrated. Chromatography 
(HE: EA = 5: 1) gave aldehyde 17 as a colorless oil $(76.0 \mathrm{mg}, 90 \%) .{ }^{1} \mathrm{H}$ NMR $(300 \mathrm{MHz}$, $\left.\mathrm{CDCl}_{3}\right) \delta$ 9.71, (s, 1H), $7.25(\mathrm{~d}, \mathrm{~J}=8.5 \mathrm{~Hz}, 2 \mathrm{H}), 6.88(\mathrm{~d}, \mathrm{~J}=8.5 \mathrm{~Hz}, 2 \mathrm{H}), 4.63(\mathrm{~d}, \mathrm{~J}=6.9$ $\mathrm{Hz}, 1 \mathrm{H}), 4.49(\mathrm{~d}, \mathrm{~J}=6.9 \mathrm{~Hz}, 1 \mathrm{H}), 4.41(\mathrm{~s}, 2 \mathrm{H}), 4.07(\mathrm{dd}, \mathrm{J}=8.6,2.6 \mathrm{~Hz}, 1 \mathrm{H}), 3.80(\mathrm{~s}$, $3 \mathrm{H}), 3.46(\mathrm{~m}, 2 \mathrm{H}), 3.22(\mathrm{~s}, 3 \mathrm{H}), 2.50(\mathrm{~m}, 1 \mathrm{H}), 1.99(\mathrm{~m}, 1 \mathrm{H}), 1.11(\mathrm{~d}, \mathrm{~J}=7.1 \mathrm{~Hz}, 3 \mathrm{H})$, $0.99(\mathrm{~d}, \mathrm{~J}=6.9 \mathrm{~Hz}, 3 \mathrm{H}) ;{ }^{13} \mathrm{C} \mathrm{NMR}\left(100 \mathrm{MHz}, \mathrm{CDCl}_{3}\right) \delta 204.3,159.4,130.7,129.5$, 114.0, 98.0, 79.2, 73.0, 71.7, 56.0, 55.5, 48.9, 36.9, 14.8, 7.1.

\section{Vinyl iodide 9.}

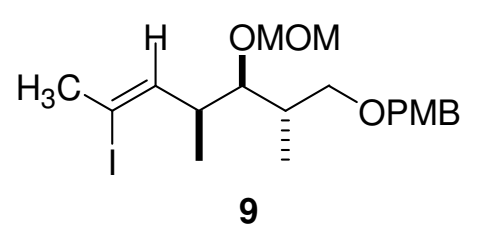

Vinyl iodide 9 was prepared as described in reference $10 \mathrm{~b}$. 